\title{
The migraine-stroke connection: A genetic perspective
}

Cephalalgia

2016, Vol. 36(7) 658-668

(C) International Headache Society 2015 Reprints and permissions: sagepub.co.uk/journalsPermissions.nav DOI: 10.1 I77/03331024I5621055 cep.sagepub.com

\author{
Rainer Malik', Bendik Winsvold ${ }^{2}$, Eva Auffenberg ${ }^{1,3}$, \\ Martin Dichgans ${ }^{1,4}$ and Tobias Freilinger ${ }^{3}$
}

\begin{abstract}
Background: A complex relationship between migraine and vascular disease has long been recognized. The pathophysiological basis underlying this correlation is incompletely understood.

Aim: The aim of this review is to focus on the migraine-vascular disorders connection from a genetic perspective, illustrating potentially shared (molecular) mechanisms.

Results: We first summarize the clinical presentation and genetic basis of CADASIL and other monogenic vascular syndromes with migraine as a prominent disease manifestation. Based on data from transgenic mouse models for familial hemiplegic migraine, we then discuss cortical spreading depression as a potential mechanistic link between migraine and ischemic stroke. Finally, we review data from genome-wide association studies, with a focus on overlapping findings with cervical artery dissection, ischemic stroke in general and cardiovascular disease.

Conclusion: A wealth of data supports a genetic link between migraine and vascular disease. Based on growing highthroughput data-sets, new genotyping techniques and in-depth phenotyping, further insights are expected for the future.
\end{abstract}

\section{Keywords}

CADASIL, migraine pathophysiology, genetic susceptibility, cortical spreading depression, hemiplegic migraine, mouse model, genomewide association study

Date received: 23 July 2015; revised: 2 November 2015; accepted: 14 November 2015

\section{Background}

Both clinical experience and epidemiological data have highlighted a complex relationship between migraine and vascular disorders, as summarized in a recent special issue of Cephalalgia (Cephalalgia 2015;35(2)). With respect to comorbidity of migraine specifically with cerebrovascular disease, meta-analyses have shown an increased risk of ischemic stroke (IS) in migraine, especially in female patients with migraine with aura (1). Further tentative evidence comes from cross-sectional studies that revealed an increased prevalence of cerebral white matter lesions and infarct-like lesions, predominantly in the posterior circulation, in migraine patients $(2,3)$.

Advancing our understanding of these aspects is highly relevant to meeting patients' needs (4) and offers the opportunity to unravel shared or overlapping pathophysiological mechanisms underlying both disorders, which in turn holds substantial translational potential.

In addition to a wealth of functional studies, with recent data suggesting the notion of migraine as a systemic vascular or endothelial disease (e.g. (5)), there is accumulating evidence for a shared genetic basis of migraine and vascular disorders. This review summarizes the most significant genetic findings relevant to the migraine-vascular disease overlap, focusing on both rare monogenic entities (cf. section 1,2), as well as more recent findings based on data from genome-wide association studies (cf. section 3).

\footnotetext{
'Institute for Stroke and Dementia Research (ISD), Munich, Germany ${ }^{2}$ FORMI and Department of Neurology, Oslo University Hospital and University of Oslo, Oslo, Norway

${ }^{3}$ Department of Neurology and Epileptology, Hertie-Institute for Clinical Brain Research (HIH), Tuebingen, Germany

${ }^{4}$ Munich Cluster of Systems Neurology (SyNergy), Germany
}

\section{Corresponding author:}

Tobias Freilinger, Abt. Neurologie mit Schwerpunkt Epileptologie, Hertie Institut für Klinische Hirnforschung, Universitätsklinikum Tübingen, Hoppe-Seyler-Str. 3, 72076 Tübingen, Germany.

Email: tobias.freilinger@uni-tuebingen.de 


\section{Monogenic vascular diseases with migraine as part of the phenotypic spectrum}

A remarkable proportion of monogenic "vascular" disease entities includes migraine or migraine-like symptoms as part of their phenotypic spectrum. The most prominent example is cerebral autosomal-dominant arteriopathy with subcortical infarcts and leukoencephalopathy (CADASIL), a cerebral small-vessel disease caused by missense mutations in the Notch 3 gene (6), which encodes a transmembrane receptor protein expressed on vascular smooth muscle cells. It is the most common form of hereditary small-vessel disease and, at the same time, the most common monogenic form of adult-onset IS (prevalence $\geq 5: 100.000$ ). Other, less common examples include retinal vasculopathy with cerebral leukodystrophy (RVCL), hereditary infantile hemiparesis, retinal arteriolar tortuosity and leukoencephalopathy (HIHRATL), hereditary hemorrhagic telangiectasia and, last but not least, mitochondrial myopathy with encephalopathy, lactic acidosis and stroke-like episodes (MELAS).

\section{CADASIL}

Migraine is an early symptom of the disease, occurring in up to $40 \%$ of patients and typically preceding other manifestations by at least a decade. Interestingly, age at onset for migraine in CADASIL patients seems to be higher than in normal migraineurs (7). In about $80 \%$ of cases, migraine manifests as migraine with aura, with a high frequency of 'atypical' (e.g. prolonged duration, hemiplegic or brainstem aura symptoms, confusion, fever or other complications) or isolated (i.e. without accompanying headache) aura symptoms (8). Interestingly, there seems to be a peak of transient neurological disturbances during gestation or in the postpartum period (9).

In subsequent stages of the disease, clinical manifestations include recurrent lacunar infarcts and cognitive deficits leading to vascular dementia, while migraine attacks mostly improve with disease progression $(10,11)$.

Magnetic resonance (MR) imaging reveals lacunar infarcts, leukoencephalopathy (typically including the external capsule as well as the temporal poles) and microbleeds (12). Diagnosis is established by direct sequencing analysis of Notch 3 and/or electron microscopic evidence of ultrastructural changes on skin biopsy (granular osmiophilic material).

The pathophysiological mechanisms underlying migraine in the setting of CADASIL are not fully understood. Vahedi and colleagues found no difference in the frequency and distribution of imaging changes between CADASIL patients with vs. without migraine (8), while Jouvent and colleagues could demonstrate morphological changes in the primary visual cortex of CADASIL patients with migraine with aura depending on the presence or absence of visual symptoms during aura (13).

To date, there is no specific treatment for CADASIL. Both migraine and ISs are treated according to current guidelines for the sporadic forms of both entities. With respect to acute migraine therapy, triptans are usually avoided due to safety concerns, although no systematic data on its risk profile in the setting of CADASIL are available; in this context, it is interesting to note that triptans were found to have good tolerability in familial hemiplegic migraine (FHM) (14), another monogenic migraine variant.

In summary, CADASIL, next to FHM (cf. below, Section 2), is the most prominent monogenic form of migraine, with migraine as a typical presenting feature in the early disease stages. A differential diagnosis of CADASIL should be considered in migraine patients (especially with the described clinical profile) with a positive family history for migraine, stroke or dementia and typical changes on MR imaging.

\section{RVCL}

Another example, which has received increasing attention more recently, is RVCL. RVCL includes disease entities previously referred to as cerebroretinal vasculopathy, hereditary vascular retinopathy or hereditary endotheliopathy, retinopathy, nephropathy and stroke. More recently, cerebral hereditary angiopathy with vascular retinopathy and impaired organ function (CHARIOT) has been introduced as a new term for RVCL.

In 2001, Ophoff and colleagues mapped the locus to chromosome $3 \mathrm{p} 21$ (15), with identification of TREXI as the causative gene in 2007 (16).

In addition to retinal vasculopathy leading to progressive visual loss, there is prominent involvement of the central nervous system, with diverse clinical manifestations, including focal neurological symptoms, cognitive deficits, depression and also migraine, notably mostly in the form of migraine without aura. Finally, there are different systemic manifestations (e.g. Raynaud's phenomenon, hepatic and renal dysfunction and gastrointestinal (GI) hemorrhage). Life expectancy in RVCL is decreased.

Regarding the association of RVCL and migraine, evidence comes primarily from a large Dutch pedigree; another characteristic clinical feature in this same family is Raynaud's syndrome, strengthening the notion of a "vascular" phenotype (17). 
Cranial magnetic resonance imaging (cMRI) shows non-contrast-enhancing T2 hyperintensities or contrast-enhancing mass lesions, mimicking a brain tumor. These "pseudo-tumors" are present in the majority of patients, often with corresponding calcifications on cranial computed tomography.

The pathophysiology of RVCL is not fully understood. TREXI has been implicated in diverse biological functions: first, it encodes a $3^{\prime}-5^{\prime}$ exonuclease (18). Secondly, it is involved in the so-called SET complex (19), which is normally associated with the endoplasmic reticulum, but upon transfer to the nucleus can induce granzyme A-mediated cell death. Finally, TREXI may be involved in cell cycle homeostasis (20). RVCLcausing mutations, by means of a frameshift, cause a Cterminally truncated protein, with subsequent disturbance of subcellular localization, while exonuclease activity remains uncompromised. The link between truncated TREX1 protein with disturbed subcellular localization and microangiopathy has not yet been deciphered.

As suggested by the clinical symptomatology (e.g. white matter changes, Raynaud's phenomenon, etc.) and also experimental data (21), an important factor in RVCL seems to be endothelial dysfunction, which may be a possible link to migraine pathophysiology. Interestingly, TREX1 mutations have also been implicated in other entities (e.g. Aicardi-Goutières syndrome, familial chilblain lupus or systemic lupus erythematosus). As opposed to RVCL, which is caused exclusively by truncating mutations, these syndromes are associated with differing types of mutations.

A transgenic mouse model for RVCL has been generated, with no published data so far (22).

\section{Other monogenic entities}

Another monogenic cerebral microangiopathy associated with migraine is HIHRATL. The disorder is caused by mutations in the COL4A1 gene, which encodes the type IV collagen alphal chain, which is present in basement membranes, including the basement membranes of the vasculature. The phenotypic spectrum is broad, including manifestations indicated by the syndrome's name, but also seizures, hemorrhagic stroke, IS and intracranial aneurysms. There is some suggestive evidence for a correlation with migraine (23).

Another monogenic entity relevant in the context of this review is hereditary hemorrhagic telangiectasia; it is caused by mutations of endoglin or activin receptor-like kinase (24). In addition to mucocutaneous telangiectasia, there are arteriovenous malformations (AVMs) affecting multiple organs, including the brain. Clinically, the disease is characterized by hemorrhages, defined by the localization of the vascular malformations. This means that patients can also suffer from hemorrhagic stroke (due to cerebral AVMs), but there is also an increased risk of IS. In addition, there is a remarkably high prevalence of migraine $(40 \%$ of patients), preferentially migraine with aura (25). Several studies have pointed out that the occurrence of migraine in hereditary hemorrhagic telangiectasia is related to the presence of pulmonary AVMs, while the significance of cerebral AVMs is more controversial (26-28); however, it should be kept in mind that the role of cerebral AVMs may have been underestimated, since they were not systematically assessed in all patients. The association between migraine and pulmonary AVMs may be due to paradoxical embolism, with cerebral microemboli triggering cortical spreading depression (CSD) events, as evidenced by a recent animal study (29). Another hypothesis would be that the pulmonary right to left shunt allows migraine-triggering agents to bypass the lung filter.

Beyond hereditary hemorrhagic telangiectasia, AVMs in general and also other structural vascular abnormalities (like in Sturge-Weber syndrome (30)) have been associated with migraine, further strengthening the migraine-vascular disease link.

Finally, MELAS combines features of both migraine and stroke. It is a multi-system disease caused by mutations in the mitochondrial DNA, mostly in the $M T$ TL1 gene, encoding tRNALeu, with the most common mutation being $\mathrm{m} \cdot 3243 \mathrm{~A}>\mathrm{G}$ (31). After unremarkable early development, age at onset is often in childhood. Early disease manifestations include generalized tonic-clonic seizures and migraine-like episodes with headache, vegetative symptoms and abdominal complaints. Subsequently, there is occurrence of recurrent episodes with acute neurological deficits (e.g. hemiparesis, aphasia and visual field defects) in the context of what is referred to as stroke-like episodes. By contrast to hemiplegic migraine, these deficits occur acutely (as opposed to gradual development in hemiplegic migraine (HM)) and are not fully reversible, since the cumulative effect of the recurrent stroke-like episodes results in increasing neurological deficits. Often, there is a sensorineuronal hearing loss.

Based on the typical clinical constellation, diagnosis is established by genetic analysis, which will in some instances require the analysis of tissues other than blood (e.g. muscle). Muscle biopsy shows ragged red fibers, and there is elevated lactate in the peripheral blood as well as in the cerebrospinal fluid (CSF). Cerebral imaging can reveal basal ganglia calcifications, focal T2-hyperintense lesions not clearly related to a vascular territory and an increasing brain atrophy. In contrast to IS, diffusion-weighted imaging will reveal an increased apparent diffusion coefficient during strokelike episodes. 


\section{Pathophysiological links between migraine and stroke in transgenic migraine animal models}

As opposed to the monogenic entities discussed above, in which migraine is part of the phenotypic spectrum, FHM is a rare monogenic disease with migraine as the predominant phenotypic feature. In fact, FHM is the monogenic form of migraine. After a brief discussion of the clinical presentation and genetic basis of FHM, we will here focus on transgenic FHM animal models, which may potentially shed light on the mechanistic correlates underlying the migraine-stroke connection, followed by a brief overview of the relevant findings from other monogenic migraine subtypes.

\section{FHM - clinical presentation and genetics}

FHM is a rare monogenic subtype of migraine with aura characterized by different degrees of transient and fully reversible hemiparesis in addition to other neurological aura symptoms (32). Otherwise, the phenotype of the aura and headache phase resemble "normal" migraine, and there is a remarkably high comorbidity with nonhemiplegic types of migraine in FHM pedigrees (33). In severe attacks, there may be various complicating features, including fever, lymphocytic pleocytosis, epileptic seizures, confusion and altered mental status up to coma (34). Further, a subset of patients, in the interval between attacks, will develop permanent neurological deficits, most commonly being a progressive cerebellar syndrome with or without cerebellar atrophy.

Genetically, FHM is heterogeneous. So far, mutations in three different genes involved in ion translocation in the central nervous system have been identified in affected pedigrees: CACNA1A (FHM1) (35), ATP1A2 (FHM2) (36) and SCN1A (FHM3) (37).

\section{FHM transgenic mouse models - the role of CSD}

Transgenic knock-in mouse models have been published for both FHM1 (mutations R192Q (38) and S218L (39)) and FHM2 (mutation W887R (40)). In addition to in-depth functional studies of these models with respect to various migraine-related aspects (for a comprehensive review, cf. (41)), the FHM1 mice have also been evaluated against the background of the "migraine-IS link".

A central pathophysiological motif in both migraine and IS seems to be CSD. CSD is a wave of an initial brief neuronal excitation, followed by a long-lasting phase of suppressed neuronal activity, travelling across the cortex at a speed of $2-3 \mathrm{~mm}$ per minute (42). As reviewed elsewhere, CSD is the likely pathophysiological correlate of the migraine aura, and experiments in FHM1 and FHM2 mice have revealed an increased susceptibility to CSD (caused by enhanced glutamatergic neurotransmission) as the neurophysiological hallmark of these animal models (38-40). At the same time, CSD is recognized also to play a central role in IS. More specifically, CSD occurs in the ischemic penumbra (referred to as "peri-infarct depolarization"), triggered by a rise in extracellular potassium (as a consequence of $\mathrm{Na}^{+} / \mathrm{K}^{+}$-ATPase failure); in this context, CSD may worsen tissue damage in the penumbra (e.g. by worsening the metabolic mismatch and/or by inducing vasoconstriction and by changing the spreading hyperemia (typical for spreading depression) to spreading ischemia) (43-45), and these processes have been shown to negatively affect infarct size and growth in animal studies (e.g. (46)). In line with these findings, spreading depression has been shown also to occur in humans with IS (47) and to be correlated with a deterioration of the clinical outcome (e.g. (45)).

Based on the significance of CSD in both stroke and migraine, Eikermann-Haerter and colleagues, in a series of studies $(48,49)$, performed an in-depth analysis of stroke vulnerability in FHM1 transgenic mouse models that were subjected to transient filament occlusion of the middle cerebral artery. The following main findings were obtained in this model: i) mutant animals showed a reduced latency for the onset of anoxic depolarization and a significantly increased frequency of peri-infarct depolarization; ii) correspondingly, MR imaging studies revealed a more rapid expansion of infarct volumes in mutant mice; iii) mutant mice were also found to have larger perfusion deficits (corresponding to the increased frequency of peri-infarct depolarization); iv) interestingly, mutated animals were found to be at a higher risk of developing ischemia in a hypo-perfused area (indicating a higher level of perfusion required for survival); v) finally, and relating to the above aspects, "clinical" outcome was worse in mutant mice (i.e. larger infarct size, neurological disability and mortality), with tentative evidence for a worse outcome in female animals.

In summary, the authors provided compelling evidence for increased stroke susceptibility in FHM1 mutant mice. This is in part related to neuronal mechanisms (i.e. hyperexcitability), as evidenced by, for example, the higher frequency of peri-infarct depolarizations. At the same time, there also seems to be a vascular mechanism, as shown by the larger perfusion deficits, which indicate inverse (i.e. vasoconstrictive) neurovascular coupling as a response to recurrent peri-infarct depolarization. As a consequence of these observations, human migraineurs may be more susceptible to suffering ischemic damage during mild ischemia, which does not cause any harm in healthy individuals. In line with this hypothesis, a study comparing diffusion-weighted MR imagine in stroke patients with vs. 
without migraine found an association of migraine with small cortical infarcts, possibly suggesting increased vulnerability to mild ischemic conditions (50).

Moreover, migraine status may negatively impact infarct growth and stroke outcome. However, this is a hypothesis that is not confirmed by clinical data so far, except for the finding of higher mortality of hemorrhagic stroke among migraineurs (51).

In a second study, Eikermann-Haerter et al. evaluated the effect of commonly prescribed migraine prophylactic drugs (e.g. topiramate or lamotrigine) in the FHM1 mouse models (and also wild-type mice), focusing specifically on its effects on stroke outcome and ischemic depolarization (49). For both drugs, an inhibitory effect on CSD had been established upon chronic treatment in rats. In different experiments, they demonstrate that daily treatment for 7 weeks with both drugs inhibits CSD and also peri-infarct depolarization and thereby leads to an improved stroke outcome both "clinically" and with respect to tissue damage; these effects required "chronic" (i.e. prophylactic) treatment and were not present after a single dose. In summary, these data, which are complimentary to the results of the initial study, reinforce the concept of CSD susceptibility as a major determinant of stroke vulnerability in FHM1 (and also wild-type) mice. This is line with data from mice with calcium channel loss-of-function mutations, which have decreased spreading depression susceptibility and accordingly smaller infarct sizes (52). Despite these highly interesting data, there are no clinical data as to whether migraine prophylactic treatment does have any influence on stroke risk in migraineurs.

In summary, the presented experimental data from these monogenic migraine/FHM mouse models suggest CSD as a plausible mechanistic link between migraine and IS.

\section{The role of CSD in other monogenic migraine variants}

The notion of a significant role for CSD is further strengthened by findings from transgenic CADASIL mouse models (both expressing a Notch3 CADASIL mutation or a Notch3 knockout mutation), which also showed an increased susceptibility to CSD - an effect present both with electrical and $\mathrm{KCl}$ stimulation for eliciting CSD (53). Interestingly, while the mechanisms underlying this hyperexcitability in a disease primarily affecting vascular smooth muscle cells still need to be elucidated, both mice were found to exhibit larger infarct sizes. With respect to the monogenic vascular entities associated with TREXI or COLAAI mutations (cf. (1)), there are no data from transgenic mouse models on their potential influence on CSD susceptibility.

\section{Outlook: other pathophysiological links between migraine and stroke}

Looking at the migraine-stroke link from a more general perspective, factors other than CSD are obviously likely also to be involved. One potential player is endothelial dysfunction, with a wealth of data highlighting its role in migraine in general. Interestingly, human studies in CADASIL patients have also suggested endothelial dysfunction in this monogenic stroke/migraine model disease (54), while this aspect has not been systematically evaluated in available CADASIL animal models, which would be an interesting target for future research.

\section{Genetic susceptibility to migraine, stroke or cardiovascular disease - findings from candidate gene studies and genome-wide association studies}

\section{Candidate gene studies}

In the pre-genome-wide association study (GWAS) era, hardly any robust genetic risk factors for migraine had been identified. However, some findings deserve attention in the context of the migraine-IS comorbidity (Table 1).

An intensively studied risk variant, for which some studies suggested an association with migraine, was the C677T polymorphism in MTHFR $(55,56)$. It is correlated with slightly elevated homocysteine levels, which may cause endothelial dysfunction and thus might potentially serve as an explanation for the increased cardiovascular morbidity in migraineurs. However, a comprehensive meta-analysis found an association with migraine only in non-Caucasian cohorts (57).

Further interesting association results are available with respect to the deletion/insertion (D/I) polymorphism in the $A C E$ gene. There was no evidence of an association of this variant with migraine, aura status or the occurrence of cardiovascular events. Interestingly, however, a significantly increased cardiovascular risk for migraine with aura patients was only present for carriers of the $\mathrm{D} / \mathrm{D}$ or $\mathrm{D} / \mathrm{I}$ genotypes (58).

\section{Findings from recent GWAS in migraine}

The migraine susceptibility genes identified through a series of large-scale GWAS of the International Headache Genetics Consortium (IHGC) in recent years (59-62) point towards several pathophysiological motifs or pathways in migraine. Next to neuronal/synaptic mechanisms, there is increasing evidence of a vascular component in migraine pathophysiology, as 
Table I. Findings from candidate gene studies and genome-wide association studies relevant to the migraine-stroke connection.

\begin{tabular}{|c|c|c|c|c|c|}
\hline Locus & Chromosome & $\begin{array}{l}\text { Migraine } \\
\text { phenotype }\end{array}$ & $\begin{array}{l}\text { Stroke } \\
\text { phenotype }\end{array}$ & Other vascular findings & References \\
\hline MTHFR & $1 p 36$ & All migraine & All stroke & - & $(55-57)$ \\
\hline$A C E$ & $17 q 23$ & All migraine & All stroke & & $(58)$ \\
\hline TGFBR2 & $3 p 24$ & All migraine & - & $\begin{array}{l}\text { Marfan syndrome, abdominal } \\
\text { aortic aneurysm }\end{array}$ & $(61,62,64-67)$ \\
\hline PHACTRI & $6 p 24$ & MO & - & CeAD, coronary artery disease & $(6 I, 62,69,7 I)$ \\
\hline$L R P I$ & $12 q 13$ & All migraine & - & Abdominal aortic aneurysm & $(60-62,72)$ \\
\hline $9 p 21$ & $9 p 21$ & MO & LAS & $\begin{array}{l}\text { Coronary artery disease, periph- } \\
\text { eral artery disease, abdominal } \\
\text { aortic aneurysm, intracranial } \\
\text { aneurysm }\end{array}$ & $(76)$ \\
\hline LMOD2-WASL & $7 q 31$ & MO & LAS & - & (76) \\
\hline
\end{tabular}

The table summarizes the findings from candidate gene as well as genome-wide association studies that are relevant to the migraine-stroke connection. The columns "Migraine phenotype" and "Stroke phenotype" indicate for which phenotypes significant association signals were established. The column "Other vascular findings" summarizes the supportive evidence for vascular disease entities other than migraine and stroke for which a correlation has been reported.

LAS: large-artery stroke; CeAD: cervical artery dissection; MO: migraine without aura.

suggested by, for example, risk variants in or near TGFBR2, PHACTR1 or LRP1 (details below Table 1), for which previous data have suggested a link with vascular phenotypes. It should be noted, however, that the evidence presented is only indirect, as is inherent to most findings from GWAS, while functional (followup) data are mostly missing. Further, the overall number of known loci is currently still reasonably low, which obviously limits a more thorough understanding of the underlying disease mechanisms as of now.

TGFBR2 encodes transforming growth factor $\beta$ receptor 2, which is involved in the regulation of cell proliferation and differentiation, as well as in extracellular matrix production (63). In line with these biological functions, mutations in TGFBR2 have been implicated in several vascular phenotypes, suggesting that a vascular component may also be involved in migraine pathophysiology. Heterozygous TGFBR2 mutations have been identified in Marfan syndrome (64), and there is some evidence for a role of TGFBR2 in abdominal aortic aneurysms (AAAs) (65). Even more interestingly, a TGFBR2 missense mutation was identified in a family with familial aortic dissection, with migraine headache present in the majority of mutation carriers (66). Expanding on these findings, another study performed complete sequencing analysis of all coding exons of TGFBR2 (and also TGFBR1) in a total of 56 cervical artery dissection (CeAD) patients, which revealed two different missense mutations (67).

The protein encoded by PHACTRI is a member of the PHACTR/scapinin family. On the one hand, it controls synaptic activity and synapse morphology, arguing in favor of neuronal/synaptic mechanisms. At the same time, there is also strong evidence for its involvement in vascular processes. PHACTRl plays a role in endothelial cell functioning (68) and has been implicated in susceptibility for early-onset myocardial infarction (MI) (69); second to IS, MI is another vascular disease entity for which comorbidity with migraine has been established in epidemiological studies (70). Interestingly, however, the MI GWAS yielded a different $P H A C T R 1$ single-nucleotide polymorphism (SNP; rs12526453), and the effect direction was opposite to that observed for migraine. More recently, and even more relevant to this topic, PHACTRl has been implicated in cervical artery dissection (CeAD) (71), which will be discussed in more detail below.

LRPI encodes the low-density lipoprotein receptorrelated protein 1 , which shows a broad expression pattern that also includes the vasculature. Among other functions, it is involved in the proliferation of vascular smooth muscle cells, building a potential link to vascular mechanisms. Interestingly, in a previous GWAS, LRPI has been identified as a risk factor for AAA (72); however, this involved a different variant (rs1466535) from the one identified in the migraine studies (rs11172113). The AAA SNP showed up in the initial phase of the recent CeAD GWAS (71), which is discussed below. A more recent study found no convincing evidence for a role of LRPI in carotid artery disease (73).

\section{New findings on migraine and $C e A D$}

There is a complex comorbidity between migraine and CeAD, as reviewed elsewhere in detail $(74,75)$. 
In summary, the key findings are: i) CeAD patients have a higher incidence of migraine compared to healthy controls; ii) patients with IS due to CeAD, when compared to non-CeAD IS patients, have a higher prevalence of migraine, which is based mostly on migraine without aura (MO); iii) as opposed to older data, the presence or absence of migraine does not significantly influence the clinical profile or outcome of CeAD.

The pathophysiology of CeAD remains elusive, with no genetic findings having been presented until recently. In 2015, however, the international Cervical Artery Dissections and Ischemic Stroke Patients (CADISP) consortium performed the first GWAS for CeAD, which included 1393 CeAD patients (recruited from the CADSIP-I and -II studies, consisting of 942 and 451 individuals, respectively) vs. 14,416 controls (71). The most promising signals $\left(p<5 \times 10^{-8}\right)$ were tested for replication in a second smaller CeAD cohort (659 cases vs. 2648 controls). Initial analysis detected two genome-wide significant signals in PHACTRI (marker rs9349379; odds ratio $(\mathrm{OR})=0.75$; $p=4.46 \times 10^{-10}$ ) and LRPl (marker rs11172113; $\mathrm{OR}=0.78 ; p=4.22 \times 10^{-8}$ ). In addition, a total of six SNPs (in five loci) had $p$-values of $<1 \times 10^{-5}$. As opposed to the $L R P 1$ variant, the PHACTR1 SNP was also significantly associated with $\mathrm{CeAD}$ in the replication cohort, with a meta-analysis of the entire data-set yielding an overall $p$-value of $1.00 \times 10^{-11}$. None of the other signals from the initial study phase were replicated, with promising findings, however, for a variant in $L N X 1$.

Against the background of the above-mentioned migraine-CeAD comorbidity, the finding with respect to PHACTRl deserves particular attention, since PHACTR 1 has been prominently implicated in genetic susceptibility to migraine. Notably, both studies identified the identical PHACTRI SNP, with the same effect direction of the minor allele. In line with epidemiological data, which emphasize a correlation between CeAD and MO (75), PHACTRI was initially identified as a migraine risk gene in a clinic-based MO cohort. Notably, the same is true for $L R P 1$, which was the second significant finding of the initial phase of the CADISP GWAS. Finally, another migraine risk variant in FHL5 (62) was found to be associated also with $\mathrm{CeAD}$ at $p=6.80 \times 10^{-4}$, again with the same effect direction.

\section{Genetic overlap between the common types of migraine and IS}

Extending on these general data from migraine GWAS, Malik and colleagues performed a more in-depth evaluation of the genetic basis of the migraine-stroke relationship, utilizing the largest currently available consortial GWAS data-sets for both IS (12,389 cases vs. 62,004 controls, METASTROKE) and migraine (23,285 cases vs. 95,425 controls, IHGC) (76). In detail, the following different approaches were used to analyze the data: first, known genome-wide significant findings for both phenotypes were tested in the respective other phenotype. Secondly, polygenic risk scores were calculated for both diseases, with subsequent testing as to whether they could predict an increased risk for the other disease; this method reflects multiple variants across the whole genome. Finally, the new method of cross-phenotype spatial mapping (CPSM), which brings specific genomic regions into focus, was used. CPSM identifies genomic windows exhibiting similar association patterns across two phenotypes using a signal processing approach, pinpointing these windows as potential overlapping risk regions. The results are summarized below.

Analysis of previously established single risk loci in the respective other sample revealed several nominally significant variants (e.g. for a major stroke risk locus on chromosome 9p21). However, these signals were not present after correction for multiple hypothesis testing, making it highly unlikely that the identical variants confer risk to both IS and migraine. Based on polygenic scores and CPSM, there was substantial genetic overlap between the two phenotypes, with stronger effects between MO and IS than between migraine with aura (MA) and IS. Furthermore, overlap was more pronounced for large-artery stroke (LAS) and cardioembolic stroke (CE) than for small-vessel disease.

The result of more pronounced overlap of MO with IS as opposed to MA with IS was somewhat surprising, given the epidemiological data that mostly indicate a higher stroke risk for patients with MA. A plausible explanation for this may be the remarkably low yield of genome-wide significant findings in MA, which may potentially be due to a role of rare variants in MA (i.e. variants not detected by GWAS). Future studies focusing on rare genetic variants may shed light on this issue. With respect to stroke subtypes and their differential relationships with migraine, similar arguments apply to small-vessel disease (SVD), for which no genomewide significant signals have been obtained so far. On the other hand, the finding of a preferential overlap of migraine with LAS and CE, which deserves attention, cannot be commented on from an epidemiological perspective, since stroke subtype-specific data are not available. With regards to stroke subtypes, looking specifically at CeAD would have been highly attractive in light of the above findings; however, this was not feasible, as CeADs were not specifically and systematically ascertained in this specific IS sample.

CPSM yielded several regions that were indicative of genetic overlap. Some of these regions had previously 
been associated with one of the two phenotypes, while others were novel findings. Some of these regions are biologically highly attractive candidates, such as the LMOD2-WASL gene region (chromosome 7q31.32), which was found for all migraine and LAS; WASL is functionally relevant for stabilizing endothelial adherens junctions.

In conclusion, for the first time, this study provides convincing evidence of shared genetic susceptibility for stroke and migraine, which will have to be dissected in more detail in future studies. In particular, it would be desirable to have precise comorbidity information in the analysed cohorts (i.e. information on IS in migraine patients and vice versa). In fact, a preliminary study from 2011 followed this approach: in the context of the Women's Health Study, a GWAS comparing migraine with vs. without cardiovascular events was performed. Five variants were found to be associated with cardiovascular events in migraineurs; however, none of them reached the level of genome-wide significance (77).

\section{Genetic overlap between the common types of migraine and coronary artery disease}

While less established than the relationship between migraine and stroke, recent evidence also suggests an approximately two-fold increased risk for coronary artery disease (CAD), including MI and angina, among patients with MA (78). This suggests a more generalized vascular involvement in migraine patients, extending beyond the cerebral vasculature. Against this background, using a similar approach as for stroke, a recent study examined the genetic overlap between migraine and CAD using two large GWAS of migraine $(19,981$ cases, 56,667 controls, IHGC) and CAD (21,076 cases, 63,014 controls, CARDIoGRAM) (79). In correspondence with the findings for stroke, there was evidence for genetic overlap between CAD and migraine, and this was restricted to $\mathrm{MO}$, while no overlap was seen with MA. Interestingly, PHACTR1 was found to exert the biggest influence on the shared risk of CAD and MO. PHACTR1, as described above, is also associated with CeAD with an effect in the same direction as for migraine, but opposite of CAD. The second strongest shared locus was close to GIP (encoding gastric inhibitory polypeptide (GIP)). GIP is involved in pancreatic insulin release and is interesting in light of reports that migraine is associated with insulin resistance and the metabolic syndrome (80). Furthermore, and against expectation, when looking at the combined effect of the shared risk variants between CAD and MO, this was found to work in opposite directions for the two disorders. In other words, patients with MO had a protective genetic risk profile for CAD. The findings suggest that while the shared genetic loci may point to shared biological mechanisms between the two disorders, they are unlikely to directly explain the observed comorbidity, and so the genetic relationship is likely to be complex.

\section{Conclusion}

In summary, the reviewed data provide a wealth of evidence for a genetic link between migraine and vascular disease. Although animal studies have suggested CSD as one potential shared motif, the pathophysiology of this relationship remains poorly understood as of now. Recently, cross-phenotype analysis of data obtained through GWAS have begun to shed light on the genetic overlap between the common types of migraine and IS or MI, providing some interesting first results and indicating the potential of this new methodology. With growing sample numbers for all analyzed phenotypes, as well as new genotyping techniques, more findings can be expected in the future. To make optimal use of the genetic data, in-depth clinical characterization of the analyzed phenotypes will be critical (e.g. focusing on specific diagnostic subcategories such as etiologic subtypes of stroke and taking into account detailed comorbidity information).

\section{Key findings}

- A remarkable proportion of monogenic "vascular" diseases include migraine or migraine-like symptoms as part of their phenotypic spectrum, with the most prominent example being CADASIL.

- Functional studies in transgenic mouse models for familial hemiplegic migraine or CADASIL suggest cortical spreading depression as a potential mechanistic link between migraine and ischemic stroke.

- Some of the migraine risk variants identified through genome-wide association studies suggest "vascular" mechanisms in migraine pathophysiology.

- Recent cross-phenotype analyses (migraine and ischemic stroke; migraine and cardiovascular disease) have indicated a shared genetic susceptibility for migraine and vascular diseases. 


\section{Funding}

The author(s) disclosed receipt of the following financial support for the research, authorship, and/or publication of this article: MD is funded by the Vascular Dementia Research Foundation. TF receives funding from the DFG (Deutsche Forschungsgemeinschaft) and the University of Tuebingen (Centre for Rare Diseases; Angewandte Klinische Forschung). BW receives funding from the Research Council of Norway (grant number 231187/F20).

\section{Declaration of conflicting interests}

The authors declared no potential conflicts of interest with respect to the research, authorship and/or publication of this article.

\section{References}

1. Kurth T, Chabriat $\mathrm{H}$ and Bousser M-G. Migraine and stroke: a complex association with clinical implications. Lancet Neurol 2012; 11(1): 92-100.

2. Kruit MC, van Buchem MA, Hofman PAM, et al. Migraine as a risk factor for subclinical brain lesions. JAMA 2004; 291(4): 427-434.

3. Palm-Meinders IH, Koppen H, Terwindt GM, et al. Structural brain changes in migraine. JAMA 2012; 308(18): 1889-1897.

4. Diener H-C, Kurth T and Holle D. Practical implications of the migraine cardio- and cerebrovascular association: unmet needs of patients. Cephalalgia 2015; 35(2): $140-145$.

5. Liman TG, Bachelier-Walenta K, Neeb L, et al. Circulating endothelial microparticles in female migraineurs with aura. Cephalalgia 2015; 35(2): 88-94.

6. Tournier-Lasserve E, Joutel A, Melki J, et al. Cerebral autosomal dominant arteriopathy with subcortical infarcts and leukoencephalopathy maps to chromosome 19q12. Nat Genet 1993; 3(3): 256-259.

7. Chabriat H, Joutel A, Dichgans M, et al. CADASIL. Lancet Neurol 2009; 8(7): 643-653.

8. Vahedi K, Chabriat H, Levy C, et al. Migraine with aura and brain magnetic resonance imaging abnormalities in patients with CADASIL. Arch Neurol 2004; 61(8): 1237-1240.

9. Roine S, Pöyhönen M, Timonen S, et al. Neurologic symptoms are common during gestation and puerperium in CADASIL. Neurology 2005; 64(8): 1441-1443.

10. Dichgans M, Mayer M, Uttner I, et al. The phenotypic spectrum of CADASIL: clinical findings in 102 cases. Ann Neurol 1998; 44(5): 731-739.

11. Opherk C, Peters N, Herzog J, et al. Long-term prognosis and causes of death in CADASIL: a retrospective study in 411 patients. Brain 2004; 127(Pt 11): 2533-2539.

12. Dichgans M, Holtmannspötter M, Herzog J, et al. Cerebral microbleeds in CADASIL: a gradient-echo magnetic resonance imaging and autopsy study. Stroke 2002; 33(1): 67-71.

13. Jouvent E, Mangin J-F, Hervé D, et al. Cortical folding influences migraine aura symptoms in CADASIL. J Neurol Neurosurg Psychiatry 2012; 83(2): 213-216.
14. Artto V, Nissilä M, Wessman M, et al. Treatment of hemiplegic migraine with triptans. Eur J Neurol 2007; 14(9): 1053-1056.

15. Ophoff RA, DeYoung J, Service SK, et al. Hereditary vascular retinopathy, cerebroretinal vasculopathy, and hereditary endotheliopathy with retinopathy, nephropathy, and stroke map to a single locus on chromosome 3p21.1-p21.3. Am J Hum Genet 2001; 69(2): 447-453.

16. Richards A, van den Maagdenberg AMJM, Jen JC, et al. C-terminal truncations in human $3^{\prime}-5^{\prime}$ DNA exonuclease TREX1 cause autosomal dominant retinal vasculopathy with cerebral leukodystrophy. Nat Genet 2007; 39(9): 1068-1070.

17. Terwindt GM, Haan J, Ophoff RA, et al. Clinical and genetic analysis of a large Dutch family with autosomal dominant vascular retinopathy, migraine and Raynaud's phenomenon. Brain 1998; 121(Pt 2): 303-316.

18. Mazur DJ and Perrino FW. Identification and expression of the TREX1 and TREX2 cDNA sequences encoding mammalian $3^{\prime}->5^{\prime}$ exonucleases. J Biol Chem 1999; 274(28): 19655-19660.

19. Chowdhury D, Beresford PJ, Zhu P, et al. The exonuclease TREX1 is in the SET complex and acts in concert with NM23-H1 to degrade DNA during granzyme A-mediated cell death. Mol Cell 2006; 23: 133-142.

20. Yang YG, Lindahl T and Barnes DE. Trex 1 exonuclease degrades ssDNA to prevent chronic checkpoint activation and autoimmune disease. Cell 2007; 131(5): 873-886.

21. Vermeersch S, Stam AH, Zielman R, et al. Trex1-mutation associated with endothelial dysfunction in RVCL patients. Cephalalgia 2011; 31(Suppl. 1): 13.

22. Klever RR, Rutten JW, Labruijere S, et al. Novel transgenic mouse models for monogenic cerebral small vessel diseases related to migraine. Cephalalgia abstract at the International Headache Congress Boston, USA. Cephalalgia 2013; 33: 954-992.

23. Lanfranconi S and Markus HS. COL4A1 mutations as a monogenic cause of cerebral small vessel disease: a systematic review. Stroke 2010; 41(8): e513-e518.

24. Faughnan ME, Palda VA, Garcia-Tsao G, et al. International guidelines for the diagnosis and management of hereditary haemorrhagic telangiectasia. J Med Genet 2011; 48(2): 73-87.

25. Marziniak M, Jung A, Guralnik V, et al. An association of migraine with hereditary haemorrhagic telangiectasia independently of pulmonary right-to-left shunts. Cephalalgia 2009; 29(1): 76-81.

26. Post MC, Letteboer TGW, Mager JJ, et al. A pulmonary right-to-left shunt in patients with hereditary hemorrhagic telangiectasia is associated with an increased prevalence of migraine. Chest 2005; 128(4): 2485-2489.

27. Post MC, van Gent MW, Plokker HW, et al. Pulmonary arteriovenous malformations associated with migraine with aura. Eur Respir J 2009; 34(4): 882-887.

28. Mawet J, Kurth T and Ayata C. Migraine and stroke: in search of shared mechanisms. Cephalalgia 2015; 35(2): 165-181.

29. Nozari A, Dileköz E, Sukhotinsky I, et al. Microemboli may link spreading depression, migraine aura, and patent foramen ovale. Ann Neurol 2010; 67(2): 221-229. 
30. Freilinger T, Peters $N$, Rémi $J$, et al. A case of Sturge-Weber syndrome with symptomatic hemiplegic migraine: clinical and multimodality imaging data during a prolonged attack. J Neurol Sci 2009; 287(1-2): 271-274.

31. Pavlakis SG, Phillips PC, DiMauro S, et al. Mitochondrial myopathy, encephalopathy, lactic acidosis, and strokelike episodes: a distinctive clinical syndrome. Ann Neurol 1984; 16(4): 481-488.

32. Headache Classification Committee of the International Headache Society (IHS). The International Classification of Headache Disorders, 3rd edition (beta version). Cephalalgia 2013; 33(9): 629-808.

33. Thomsen LL, Eriksen MK, Roemer SF, et al. A population-based study of familial hemiplegic migraine suggests revised diagnostic criteria. Brain 2002; 125(Pt 6): 1379-1391.

34. Ducros A, Denier C, Joutel A, et al. The clinical spectrum of familial hemiplegic migraine associated with mutations in a neuronal calcium channel. $N$ Engl J Med 2001; 345(1): 17-24.

35. Ophoff RA, Terwindt GM, Vergouwe MN, et al. Familial hemiplegic migraine and episodic ataxia type- 2 are caused by mutations in the $\mathrm{Ca}^{2+}$ channel gene CACNL1A4. Cell 1996; 87(3): 543-552.

36. Fusco MD, Marconi R, Silvestri L, et al. Haploinsufficiency of ATP1A2 encoding the $\mathrm{Na}^{+} / \mathrm{K}^{+}$pump $\alpha 2$ subunit associated with familial hemiplegic migraine type 2. Nat Genet 2003; 33(2): 192-196.

37. Dichgans M, Freilinger T, Eckstein G, et al. Mutation in the neuronal voltage-gated sodium channel SCN1A in familial hemiplegic migraine. Lancet 2005; 366(9483): 371-377.

38. van den Maagdenberg AMJM, Pietrobon D, Pizzorusso $\mathrm{T}$, et al. A Cacnala knockin migraine mouse model with increased susceptibility to cortical spreading depression. Neuron 2004; 41(5): 701-710.

39. van den Maagdenberg AMJM, Pizzorusso T, Kaja S, et al. High cortical spreading depression susceptibility and migraine-associated symptoms in $\mathrm{Ca} \mathrm{v} 2.1 \mathrm{~S} 218 \mathrm{~L}$ mice. Ann Neurol 2010; 67(1): 85-98.

40. Leo L, Gherardini L, Barone V, et al. Increased susceptibility to cortical spreading depression in the mouse model of familial hemiplegic migraine type 2. PLoS Genet 2011; 7(6): e1002129.

41. Ferrari MD, Klever RR, Terwindt GM, et al. Migraine pathophysiology: lessons from mouse models and human genetics. Lancet Neurol 2015; 14(1): 65-80.

42. Lauritzen M. Pathophysiology of the migraine aura. The spreading depression theory. Brain 1994; 117(Pt 1): 199-210.

43. Strong AJ, Anderson PJ, Watts HR, et al. Peri-infarct depolarizations lead to loss of perfusion in ischaemic gyrencephalic cerebral cortex. Brain 2007; 130(Pt 4): 995-1008.

44. Shin HK, Dunn AK, Jones PB, et al. Vasoconstrictive neurovascular coupling during focal ischemic depolarizations. J Cereb Blood Flow Metab 2006; 26(8): 1018-1030.

45. Dreier JP, Woitzik J, Fabricius M, et al. Delayed ischaemic neurological deficits after subarachnoid haemorrhage are associated with clusters of spreading depolarizations. Brain 2006; 129(Pt 12): 3224-3237.

46. Lückl J, Dreier JP, Szabados T, et al. Peri-infarct flow transients predict outcome in rat focal brain ischemia. Neuroscience 2012; 226: 197-207.

47. Nakamura H, Strong AJ, Dohmen C, et al. Spreading depolarizations cycle around and enlarge focal ischaemic brain lesions. Brain 2010; 133(Pt 7): 1994-2006.

48. Eikermann-Haerter K, Lee JH, Yuzawa I, et al. Migraine mutations increase stroke vulnerability by facilitating ischemic depolarizations. Circulation 2012; 125(2): 335-345.

49. Eikermann-Haerter K, Lee JH, Yalcin N, et al. Migraine prophylaxis, ischemic depolarizations, and stroke outcomes in mice. Stroke 2015; 46(1): 229-236.

50. Øygarden H, Kvistad CE, Bjørk M, et al. Diffusionweighted lesions in acute ischaemic stroke patients with migraine. Acta Neurol Scand Suppl 2014; 198: 41-46.

51. Kurth T, Kase CS, Schürks M, et al. Migraine and risk of haemorrhagic stroke in women: prospective cohort study. BMJ 2010; 341: c3659.

52. Tian X, Zhou Y, Gao L, et al. Analysis of ischemic neuronal injury in Cav2.1 channel $\alpha 1$ subunit mutant mice. Biochem Biophys Res Commun 2013; 434(1): 60-64.

53. Eikermann-Haerter K, Yuzawa I, Dileköz E, et al. Cerebral autosomal dominant arteriopathy with subcortical infarcts and leukoencephalopathy syndrome mutations increase susceptibility to spreading depression. Ann Neurol 2011; 69(2): 413-418.

54. Peters N, Freilinger T, Opherk C, et al. Enhanced L-arginine-induced vasoreactivity suggests endothelial dysfunction in CADASIL. J Neurol 2008; 255(8): 1203-1208.

55. Rubino E, Ferrero M, Rainero I, et al. Association of the C677T polymorphism in the MTHFR gene with migraine: a meta-analysis. Cephalalgia 2009; 29(8): $818-825$.

56. Oterino A, Toriello M, Valle N, et al. The relationship between homocysteine and genes of folate-related enzymes in migraine patients. Headache 2010; 50(1): 99-168.

57. Schürks M, Rist PM and Kurth T. MTHFR677C $>$ T and ACED/I polymorphisms in migraine: a systematic review and meta-analysis. Headache 2010; 50(4): 588-599.

58. Schurks M, Zee RYL, Buring JE, et al. ACE D/I polymorphism, migraine, and cardiovascular disease in women. Neurology 2009; 72(7): 650-656.

59. Anttila V, Stefansson H, Kallela M, et al. Genome-wide association study of migraine implicates a common susceptibility variant on 8q22.1. Nat Genet 2010; 42(10): 869-873.

60. Chasman DI, Schürks M, Anttila V, et al. Genome-wide association study reveals three susceptibility loci for common migraine in the general population. Nat Genet 2011; 43(7): 695-698.

61. Freilinger T, Anttila V, de Vries B, et al. Genome-wide association analysis identifies susceptibility loci for migraine without aura. Nat Genet 2012; 44(7): 777-782.

62. Anttila V, Winsvold BS, Gormley P, et al. Genome-wide meta-analysis identifies new susceptibility loci for migraine. Nat Genet 2013; 45(8): 912-917. 
63. Lin HY, Wang XF, Ng-Eaton E, et al. Expression cloning of the TGF-beta type II receptor, a functional transmembrane serine/threonine kinase. Cell 1992; 68(4): 775-785.

64. Mizuguchi T, Collod-Beroud G, Akiyama T, et al. Heterozygous TGFBR2 mutations in Marfan syndrome. Nat Genet 2004; 36(8): 855-860.

65. Biros E, Walker PJ, Nataatmadja M, et al. Downregulation of transforming growth factor, beta receptor 2 and Notch signaling pathway in human abdominal aortic aneurysm. Atherosclerosis 2012; 221(2): 383-386.

66. Law C, Bunyan D, Castle B, et al. Clinical features in a family with an $\mathrm{R} 460 \mathrm{H}$ mutation in transforming growth factor receptor 2 gene. J Med Genet 2006; 43(12): 908-916.

67. Pezzini A, Drera B, Del Zotto E, et al. Mutations in TGFBR2 gene cause spontaneous cervical artery dissection. J Neurol Neurosurg Psychiatry 2011; 82(12): 1372-1374.

68. Jarray R, Allain B, Borriello L, et al. Depletion of the novel protein PHACTR-1 from human endothelial cells abolishes tube formation and induces cell death receptor apoptosis. Biochimie 2011; 93(10): 1668-1675.

69. Kathiresan S, Voight BF, Purcell S, et al. Genome-wide association of early-onset myocardial infarction with single nucleotide polymorphisms and copy number variants. Nat Genet 2009; 41(3): 334-341.

70. Bigal ME, Kurth T, Santanello N, et al. Migraine and cardiovascular disease: a population-based study. Neurology 2010; 74(8): 628-635.

71. Debette S, Kamatani Y, Metso TM, et al. Common variation in PHACTR1 is associated with susceptibility to cervical artery dissection. Nat Genet 2015; 47(1): 78-83.
72. Bown MJ, Jones GT, Harrison SC, et al. Abdominal aortic aneurysm is associated with a variant in low-density lipoprotein receptor-related protein 1. Am J Hum Genet 2011; 89(5): 619-627.

73. Giusti B, Galora S, Saracini C, et al. Role of rs 1466535 low density lipoprotein receptor-related protein 1 (LRP1) gene polymorphism in carotid artery disease. Atherosclerosis 2014; 237(1): 135-137.

74. Rist PM, Diener H-C, Kurth T, et al. Migraine, migraine aura, and cervical artery dissection: a systematic review and meta-analysis. Cephalalgia 2011; 31(8): 886-896.

75. Metso TM, Tatlisumak T, Debette $S$, et al. Migraine in cervical artery dissection and ischemic stroke patients. Neurology 2012; 78(16): 1221-1228.

76. Malik R, Freilinger T, Winsvold BS, et al. Shared genetic basis for migraine and ischemic stroke: a genome-wide analysis of common variants. Neurology 2015; 84(21): 2132-2145.

77. Schürks M, Buring JE, Ridker PM, et al. Genetic determinants of cardiovascular events among women with migraine: a genome-wide association study. PLoS ONE 2011; 6(7): e22106.

78. Sacco S, Ornello R, Ripa P, et al. Migraine and risk of ischaemic heart disease: a systematic review and metaanalysis of observational studies. Eur J Neurol 2015; 22(6): 1001-1011.

79. Winsvold BS, Nelson CP, Malik R, et al. Genetic analysis for a shared biological basis between migraine and coronary artery disease. Neurol Genet 2015; 1: e10.

80. Winsvold BS, Sandven I, Hagen K, et al. Migraine, headache and development of metabolic syndrome: an 11-year follow-up in the Nord-Trøndelag Health Study (HUNT). Pain 2013; 154(8): 1305-1311. 